\title{
NUMERICAL SIMULATION FOR RANDOM AEROELASTIC RESPONSES USING INVERSE FOURIER TRANSFORM
}

\author{
Tetsuhiko UEDA ${ }^{1}$, Kenichi SAITOH ${ }^{2}$ \\ ${ }^{1}$ Department of Aerospace Engineering, Nagoya University, Furocho, 464-8603, Nagoya, Japan \\ ${ }^{2}$ Institute of Aeronautical Technology, Japan Aerospace Exploration Agency (JAXA), \\ Osawa, 181-0015, Mitaka, Japan \\ E-mails: ${ }^{1}$ ueda@nagoya-u.jp (corresponding author); ${ }^{2}$ ksaitoh@chofu.jaxa.jp
}

Received 08 September 2014; accepted 02 May 2016

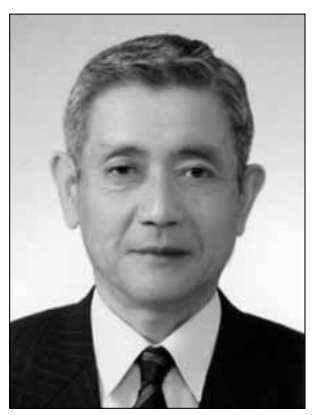

Tetsuhiko UEDA, $D r$

Education: BSc (1967-1971), MSc (1971-1973), Dr Eng. (1973-1976), Aeronautics, University of Tokyo.

Affiliations and functions: National Aerospace Laboratory (1976-2003), Director, Aviation Safety and Technology Center, JAXA (2003-2004), Professor, Nagoya University (20042012), Director, Composite Engineering Research Center (2009-2012).

Experience: Visiting Scholar, Princeton University (1980-1982), Invited Researcher, NRC Canada (1988-1989), ALFLEX Project (1993-1996), President of the Japan Society for Aeronautical and Space Sciences (2006).

Present position: Professor Emeritus-Nagoya University, Consulting Fellow-JAXA.

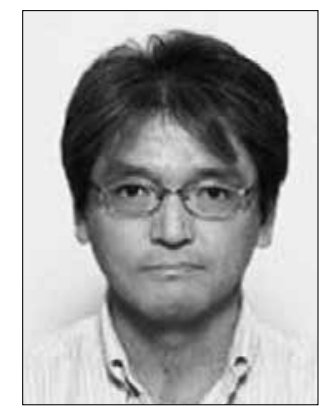

Kenichi SAITOH, $\mathrm{Dr}$

Education: BSc (1987-1991), MSc (1991-1993), Dr Eng. - 2012, Aerospace Engineering, Nagoya University.

Affiliations and functions: National Aerospace Laboratory (1993-2003), Japan Aerospace Exploration Agency (JAXA) (2003 - to present.)

Experience: Visiting Researcher, German Aerospace Laboratory-DLR (2003-2004).

Present position: Associate Senior Researcher, Structures and Advanced Composite Research Unit, Institute of Aeronautical Technology, JAXA.

\begin{abstract}
This paper reports a new simulation technique for an aeroelastic system which responds to random external forces. Since the aeroelastic system including the effects of unsteady aerodynamics is ordinarily described in the frequency domain, the Inverse Discrete Fourier Transform (IDFT) can be utilized to simulate its random response. The response caused by the external random noise is calculated through a transfer function first in the frequency domain and then converted to the time domain. The objective of the present study is to provide mathematical time history data for evaluating the various estimation methods of the flutter boundary from subcritical responses in flight and/or wind tunnel testing. An example application to the method of flutter prediction is shown. The technique can also be used to evaluate the effects of the active control device coping with atmospheric turbulence.
\end{abstract}

Keywords: aeroelasticity, random response, numerical simulation, Fourier transform, flutter prediction, IDFT.

\section{Introduction}

Wing flutter is one of the most critical problems to be solved before the final stage of aircraft design. After the design procedure, it must be demonstrated by flight tests that the airplane be completely free from fluttering. Usually, flutter tests are first conducted by using scaled models to find the flutter boundaries in the wind tunnel. In the following actual flight tests, it will be of importance to estimate the flutter boundary from the subcritical response data within the flight envelope. Even during the wind tunnel test, we sometimes lose precious wing models by an abrupt occurrence of fluttering. 
Therefore, in both cases, the reliable prediction of the critical speed before the flutter onset is highly required. Although various methods have been proposed, it is still difficult to predict flutter. It is also difficult even to evaluate prediction methods because obtaining data by experiments and/or by analyses is not an easy task. The analysis includes the complicated calculation of unsteady aerodynamic forces for the response of the aeroelastic systems. As for finding the flutter boundary, the method is thought to be matured with the aid of the linear theory of unsteady lifting surfaces. There is no efficient method, however, for simulating the subcritical response, since an unsteady aerodynamics is provided in the frequency domain. The other reason for the lack of a reliable method for prediction is that it is definitely difficult to obtain subcritical response data together with the actual flutter occurrence experimentally. Hence, the analytical simulation with random external loads and/or with random internal noise of instruments is requisite.

The phenomenon of flutter involves the unsteady aerodynamic forces which are induced by the wing motion itself. In order to analyse it, theoretical aerodynamic forces are provided with the functions of the so-called reduced frequency which is non-dimensionalized by a flow speed and a representative length. In the practical calculation, these forces are computed with twenty reduced frequencies at most for several deflection modes with the aid of the unsteady lifting surface theory. Values between frequencies are approximated by the interpolation technique to reduce cumbersome calculations of the generalized forces solving the singular integral equation.

Nowadays, however, the performance of an electric computer has progressed tremendously and has made it easy to calculate the unsteady aerodynamic forces even using a small workstation. Therefore, it has become feasible to compute them for literally thousands of frequencies. This leads us to an idea that the time response of an aeroelastic system can be simulated by using thousands of digital data in the frequency domain through the Inverse Discrete Fourier Transform (IDFT). Furthermore, if we choose the number of data, say 1024 or 2048, for example, we can utilize the technique of the FFT directly, even for the inverse transform without any approximation of interpolation between frequencies. This enables us to obtain the digital data of the response in time with a constant flow speed below the flutter from the analysis in the frequency domain.

This paper reports in detail the data handling of that conversion to simulate the aeroelastic response. It assumes that the response is caused by random turbulence in the present report.

The results can be applied to evaluate the reliability of various flutter prediction methods and to find the proper location of sensors, which depends on the flutter characteristics. The simulation technique is also expected to contribute to examining the active control effects to attenuate the gust load.

\section{Discrete Fourier Transform (DFT)}

The discrete Fourier transform and its inverse (Randall 1977) are defined by the following pair of equations:

$$
\begin{aligned}
& G(m)=\frac{1}{N} \sum_{n=0}^{N-1} g(n) \mathrm{e}^{-i \frac{2 \pi m n}{N},} \\
& g(n)=\sum_{m=0}^{N-1} G(m) \mathrm{e}^{i \frac{2 \pi m n}{N},}
\end{aligned}
$$

where $g(n)$ denotes a series of digital signal equally sampled in the time domain and $G(m)$ DFT. Equation (2) is the inverse discrete Fourier transform which is abbreviated as the IDFT. The symbols $m$ and $n$ are integers and $N$ selected as the multiple powers of 2 , so as to utilize the efficient Fast Fourier Transform technique by Cooley and Tukey. Mathematically, the DFT is based on the assumption that the time signal is periodic. In practical cases, however, this restriction causes no problems because of the finite duration of the actual signal.

\section{Impulsive response and the transfer function}

Generally, the time history of the response can be regarded as a convolution integral of an impulsive response function and the external loads. An impulsive response function is equivalent to the inverse of the Laplace transform of the transfer function itself (Thomson 1960). Therefore, it can be calculated with the inverse Fourier transform when the frequency response function of the system is known.

For a continuous signal, the impulsive response function of a system is directly related to the inverse Laplace transform of a transfer function, $H(s)$ :

$$
G(t)=\mathcal{L}^{-1}[H(s)]
$$

In the Laplace transformed domain, the impulse as an external force can be given by a unit function. Then the response becomes:

$$
G(s)=H(s) \cdot 1 \text {. }
$$

If we think that the steady state is a response by the harmonic excitation, Eq.(4), by putting $s=i \omega$, yields:

$$
G(i \omega)=H(i \omega) \cdot 1 \text {. }
$$

This relationship describes the response with the uniformly distributed exciting force in the frequency domain. On the other hand, the definition of the Laplace transform is given by:

$$
G(s)=\int_{0}^{\infty} g(t) e^{-s t} d t .
$$


Putting $s=i \omega$ in Eq. (6) leads us to an expression for the steady state response by harmonic excitation:

$$
G(i \omega)=\int_{0}^{\infty} g(t) e^{-i \omega t} d t .
$$

Here, in order to clarify the relationship between the Laplace transform and the Fourier transform, we extend the impulsive response function to the negative region of time:

$$
g(-t)=g(t),(t>0) \text {. }
$$

Then, the corresponding part of the function in the frequency domain becomes:

$$
\begin{aligned}
& G(-i \omega)=\overline{G(i \omega)}=\int_{0}^{\infty} g(t) \mathrm{e}^{i \omega t} d t= \\
& \int_{-\infty}^{0} g(-t) \mathrm{e}^{-i \omega t} d t=\int_{-\infty}^{0} g(t) \mathrm{e}^{-i \omega t} d t
\end{aligned}
$$

Thus, we obtain the Fourier transform as:

$$
G(i \omega)+\overline{G(i \omega)}=\int_{-\infty}^{\infty} g(t) \mathrm{e}^{-i \omega t} d t=\hat{g}(\omega) .
$$

If we assume the transfer function $G(s)$ for an aeroelastic system including the effects of unsteady forces, then its frequency response function of the system becomes:

$$
G(i \omega)=\hat{g}(\omega)
$$

This enables us to write the impulsive response $g(t)$ as an inverse Fourier transform.

$$
g(t)=\mathcal{F}^{-1}[G(i \omega],(t>0) .
$$

Equation (12) implies that the discrete data of the time history can be converted from the discrete frequency data and vice versa through the relations of Eqs (1) and (2).

It should be noted here that the amplitudes of the response calculated form the transfer function in the frequency domain by using the digital data with finite values are different from those obtained with a unit impulse in the continuous time domain. We have to adjust the level of each input whenever we want to compare those results.

Once the impulsive response function of a system has been obtained, then the time history for arbitrary external forces can be generated by the convolution integral (Nishijima et al. 2009). There is an alternative method to obtain the response in the time domain, i.e. the application of the inverse Fourier transform after the multiplication of the transfer function and the Fourier transform of the external forces. The present paper introduces the latter method, since it is more efficient for simulating the response.

\section{Processing of digital signals}

The Theodorsen function for a two dimensional airfoil or the unsteady lifting surface theory (Küssner 1940) for a finite wing provide the unsteady aerodynamic forces due to the system motion as functions of the reduced frequency, i.e. in the frequency domain. Therefore, they cannot be expressed with the Laplace operator $s$ which corresponds to the differentiation with respect to time. This means that the transfer function of an aerodynamic system is written with the matrix of complex numbers while its response of Eq. (2) has real numbers. When applying the IDFT to obtain time history data, we have to take this condition into account carefully. The procedure is described as follows.

(1) Assume the $G(m)$ as the aeroelastic response function in the frequency domain and calculate $(N / 2+1)$, values with an interval $\Delta \omega$ from $\omega=0$ to $\omega=(N / 2) \Delta \omega$.

(2) The corresponding duration time (a theoretical periodic interval in the time domain) and the sampling period of the digital signal become, respectively:

$\mathrm{T}=2 \pi / \Delta \omega$ and $\Delta t=T / N$.

(3) In order to hold the causality, the following complex conjugate values are allotted for $G(m)$ with $m=(N / 2+1), \ldots,(N-1)$ :

$\mathrm{G}(m)=\operatorname{Conj}[G(N-m)]$.

(4) For the value on the middle folding point, we enforce:

$$
\operatorname{Im}[G(N / 2)]=0 .
$$

(5) Since the present simulation is for the aeroelastic subcritical response, the static deformation is not necessary. Hence,

$$
G(0)=0 \text {. }
$$

(6) Application of the IDFT to $G(0), \ldots, G(N-1)$, as formed, yields a time history consisting of an $N$ number of digital data.

\section{Governing equation of an aeroelastic system}

Assuming $D(i \omega)$ as the impedance matrix of a mechanical system and $A(\omega)$ as the unsteady aerodynamic matrix, we can write the governing equation for an aeroelastic system with the generalized coordinates $\mathbf{q}$ as:

$$
[D(\mathrm{~s})+A(\omega)] \mathbf{q}=\mathrm{f},
$$

where the $f$ in the right hand side of the equation denotes the generalized external force, which will be a random aerodynamic noise in the present case. If we re-denote the transfer function of the system in the frequency domain as $H(\omega)$, then it can be obtained from Eq. (17) as:

$$
H(\omega)=[D(\mathrm{i} \omega)+A(\omega)]^{-1} \text {. }
$$

Then the impulsive response of the system, including the effects of the unsteady aerodynamic forces, is written for each component as:

$$
H_{i j}(t)=\mathcal{F}^{-1}\left[H_{i j}(\omega)\right],(t>0) .
$$




\section{A typical section airfoil}

As an example problem, we shall use a typical section of a two-dimensional airfoil, shown in Figure 1.

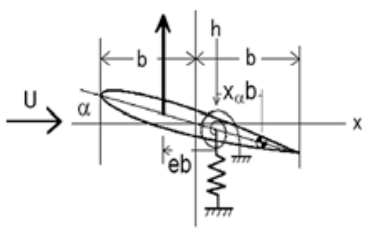

Fig. 1. Typical section airfoil

Each parameter is defined in the non-dimensionalized form. Unlike the flutter analysis, the frequency $\Omega$ is normalized with respect to the pitching frequency $\omega_{\alpha}$ instead of the so-called reduced frequency. Defining the generalized coordinate vector by $\{h, \alpha\}^{\mathrm{T}}$, we obtain a part of the transfer function excluding aerodynamic forces as:

$$
D(s)=s^{2}\left[\begin{array}{cc}
1 & x_{\alpha} \\
x_{\alpha} & r_{\alpha}^{2}
\end{array}\right]+\left[\begin{array}{cc}
R^{2} & 0 \\
0 & r_{\alpha}^{2}
\end{array}\right],
$$

where $R$ is the frequency ratio and $r_{\alpha}^{2}$ is the moment of inertia of the section which has been non-dimensionalized by the representative length and mass.

The work by Bisplinghoff et al. (1955) provides a two-dimensional incompressible unsteady aerodynamic matrix for Eq. (17) with the Theodorsen function having the argument of reduced frequency. We denote the mass ratio as $\mu$ and the non-dimensional speed $U^{*}=U /\left(b \omega_{\alpha}\right)$. Additionally, the non-dimensional dynamic pressure and the time are introduced by $Q=2 U^{* 2} / \mu$ and $t *=\omega_{\alpha} t$, respectively. As the flow is fixed to a certain speed in the present case, the Theodorsen function $C(k)=C\left(\Omega / U^{*}\right)$ can be rewritten as $C(\Omega)$. Then the aerodynamic matrix can be given by:

$$
\begin{aligned}
& \mu A(\Omega)=-\Omega^{2}\left[\begin{array}{cc}
1 & -e+\frac{1}{2} \\
-e+\frac{1}{2} & e^{2}-e+\frac{3}{8}
\end{array}\right]+ \\
& i \Omega U^{\star}\left[\begin{array}{cc}
2 C(\Omega) & 1+2(1-e) C(\Omega) \\
-2 e C(\Omega) & (1-e)-2 e(1-e) C(\Omega)
\end{array}\right]+ \\
& U^{\star 2} C(\Omega)\left[\begin{array}{cc}
0 & 2 \\
0 & -2 e
\end{array}\right] .
\end{aligned}
$$

In the following numerical examples, the parameters of the system the same parameters as for the case(n) on the P538 of the reference are selected. Those are $\mu=10, \quad e=0.2, \quad x_{\alpha}=0.1, \quad r_{\alpha}^{2}=0.25, \quad$ and $R=0.3$.

This combination of parameters results in the flutter critical dynamic pressure result of $Q_{F}=0.80$ with the frequency $\Omega_{F}=0.62$.

\section{Finite state model}

In order to compare the results obtained by the present IDFT procedure, a time domain method using the finite state model is introduced. The aerodynamic effect is embedded in the system of differential equations approximately with the augmented state. For unsteady aerodynamic terms, the following form of the finite state (Baldelli et al. 1995) is used:

$$
F_{a}(s, \mathbf{q})=\left(A_{2} s^{2}+A_{1} s+A_{0}+\sum_{i=1}^{3} \frac{A_{L_{i}}}{s+\lambda_{i}}\right) \mathbf{q},
$$

where the symbol q denotes the generalized coordinate vector. The coefficients in Eq. (22), $A_{2}, A_{1}, A_{0}$, and $A_{L_{i}}$ are determined by using the least square method accompanied with the calculation of the DPM (Ueda 1983) for the frequencies from 0.01 to 2.0. The interval of the frequency is selected as 0.01 and for the three arbitrary parameters $\lambda_{i}$ s as $0.1,0.5$, and 1.5.

\section{Numerical examples}

\subsection{Impulsive response}

For a certain dynamic pressure below the critical speed, the impulsive response is calculated by applying the IDFT to the transfer function which is given by Eq. (18). The discrete values of the function are computed for $N / 2=1024$ frequencies with a frequency increment, $\Delta \Omega=0.01$. This corresponds to 2048 data in the time domain with the sampling rate, $\Delta t^{*}=0.3068$. In the practical calculation, this distribution may be appropriately cut for higher frequencies above $\Omega=5$, since the two natural frequencies in this case are $\Omega=0.3$ and 1 . Figure 2 illustrates the response of $h\left(t^{*}\right)=h_{\alpha h}+h_{\alpha \alpha}$ due to the $\alpha$ impulse.

It should be noted that a unit impulse, which is Dirac's delta function mathematically in the continuous space, corresponds to a finite value of $1 / \Delta t$ solely at the starting point of the discrete data series and that the constant amplitude in the frequency domain must be $1 /(N \Delta t)$ to make each signal level of power the same. It can be seen from the figures that the results obtained by the IDFT agree well with those obtained by the finite state model in the time domain.

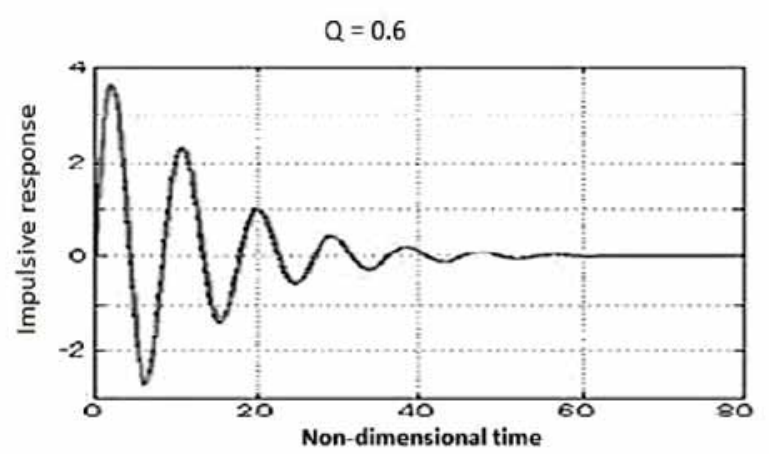

(a) Impulsive response by the finite state model 

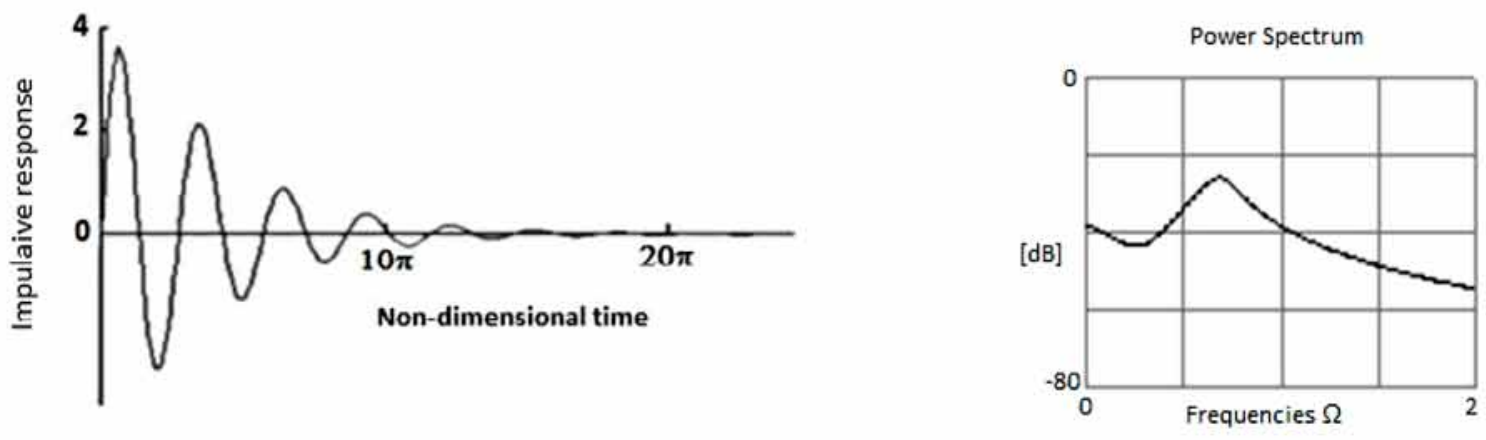

(b) Impulsive response by the inverse Fourier transform

Fig. 2. Comparison of methods

\subsection{Random noise}

Random noise can be generated by the present method as follows. First, let us assume the transfer function of Eq. (18) as a unit matrix. Then, the components of random external forces in Eq. (17) are calculated in the frequency domain with:

$$
F(m)=\Phi(m) \mathrm{e}^{i \varphi_{m}},
$$

where the phase $\varphi_{m}$ is provided with a uniformly distributed random number between 0 and $2 \pi$. In the case of the white Gaussian noise, the spectrum $\Phi(m)$ should be the Gaussian distribution. The IDFT conversion after these calculations gives a series of random signal. Similar noise can also be generated in the time domain by superimposing the cosine function in the entire interval (Shinozuka, Jan 1972). Results by both methods are compared in Figure 3 for the series of random signal having the unit average amplitude and the standard deviation of 0.3 .

The results agree well with each other as both procedures are theoretically equivalent. It should be noted that the present method is much more efficient due to the FFT algorithm in terms of computation time.
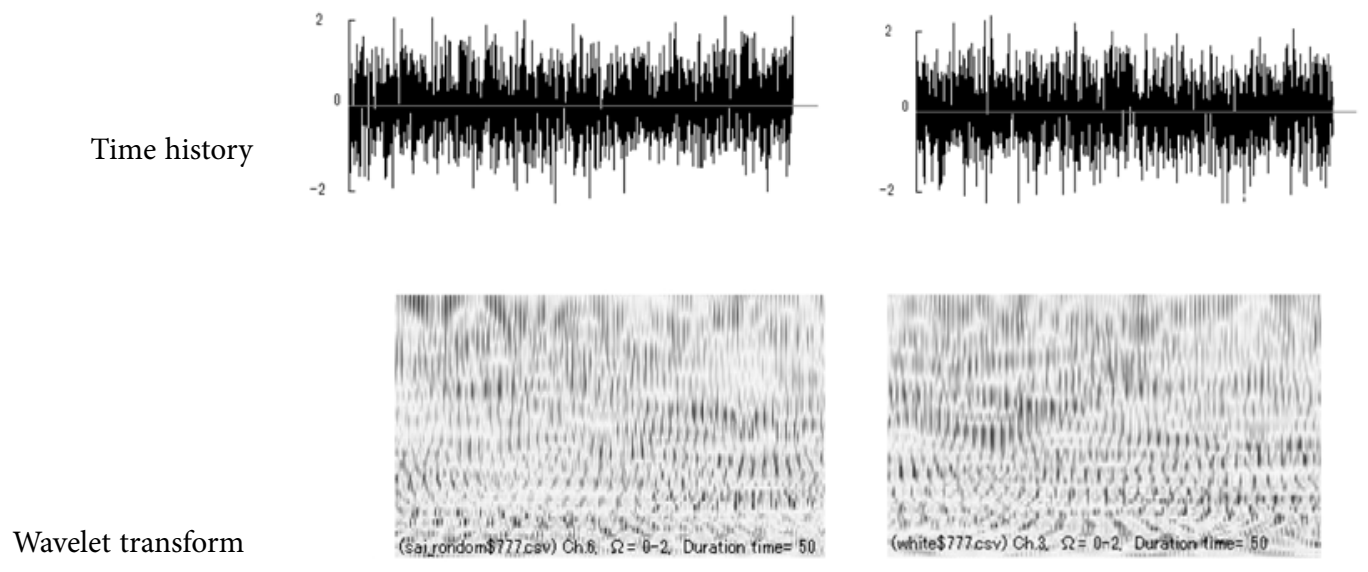

Power spectrum

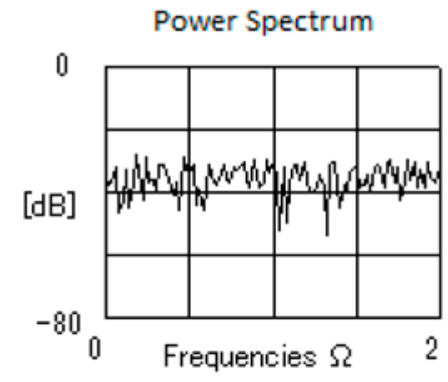

a)

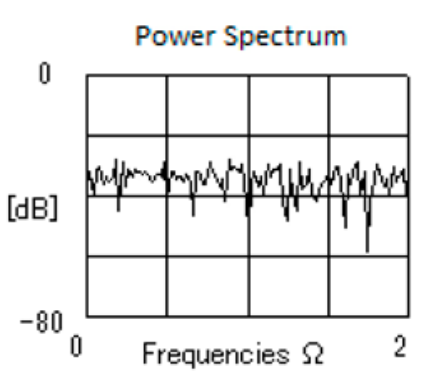

b)

Fig. 3. (a) Time Domain Method, (b) Present IDFT Method 


\subsection{Simulation of random response of a typical section airfoil}

For the same dynamic pressure as that in the example in 8.1, an aeroelastic response due to a random noise has been calculated. The results are depicted in Figure 4(a). Figure 4(b) shows its power spectrum. The smooth curve in the figure corresponds to the power spectrum of the impulsive response, i.e. the response without noise. It can be seen that the randomness is properly included in the response.

Furthermore, the dependency on the dynamic pressure for the cross spectrum between the $h$ and $\alpha$ in the response is illustrated in Figure 4(c). The figure reveals the coupling of the two modes going into flutter at the dynamic pressure of $\mathrm{Q}=0.8$.

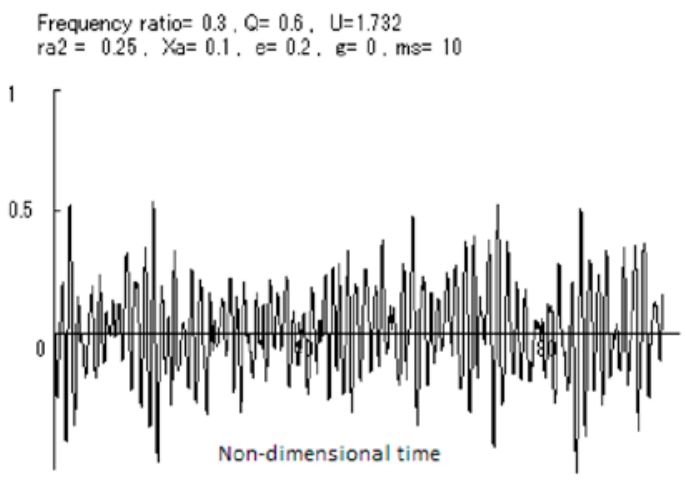

(a) Time history of random response $(\mathrm{Q}=0.6)$

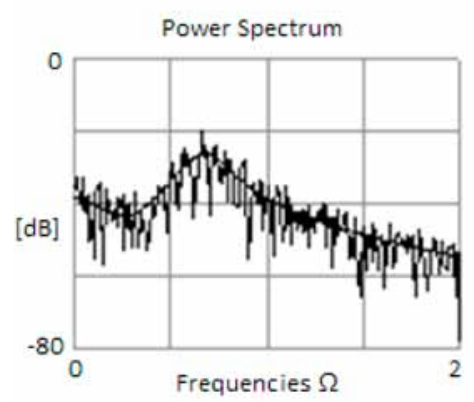

(b) Power spectrum

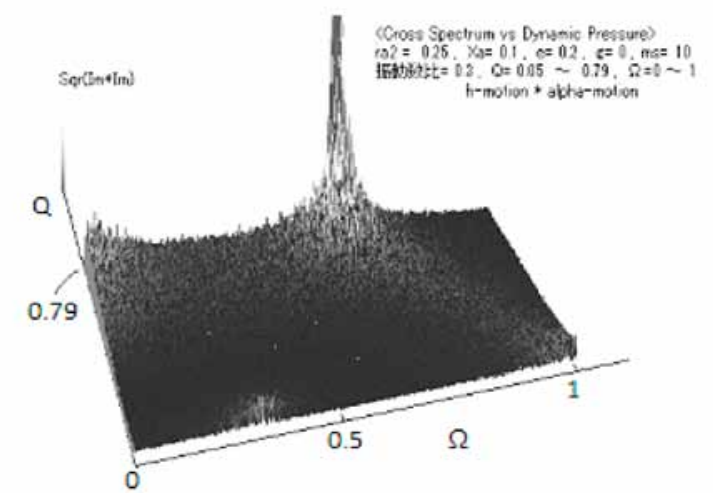

(c) Dependency of cross spectra on Q

Fig. 4. Results of simulation

\section{Application of the IDFT simulation method}

An example application of the present technique has been carried out on the flutter prediction using the wavelet transform (Ueda et al. 2009). The definition of the wavelet is given by:

$$
\begin{aligned}
& f_{\psi}^{W}(b, a)=\frac{1}{\sqrt{|a|}} \int_{-\infty}^{\infty} f(t) \overline{\psi\left(\frac{t-b}{a}\right)} d t, \\
& \psi(t)=\frac{1}{\sqrt{2 \pi \sigma^{2}}} \exp \left(-\frac{t^{2}}{2 \sigma^{2}}\right) \exp \left(i \omega_{0} t\right) .
\end{aligned}
$$

Here Gabor's mother wavelet was used as described in Eq. (25).

In Figure 5, the simulated response signals of the $\alpha$ motion are displayed for several dynamic pressures. They can be deemed as the output of a "virtual experiment" for the purpose of examining flutter prediction methods. These data have been applied to the wavelet prediction method. The result is shown in Figure 6.

It can be seen from the figure that the present simulation technique provides a reasonable output of the virtual experiment.
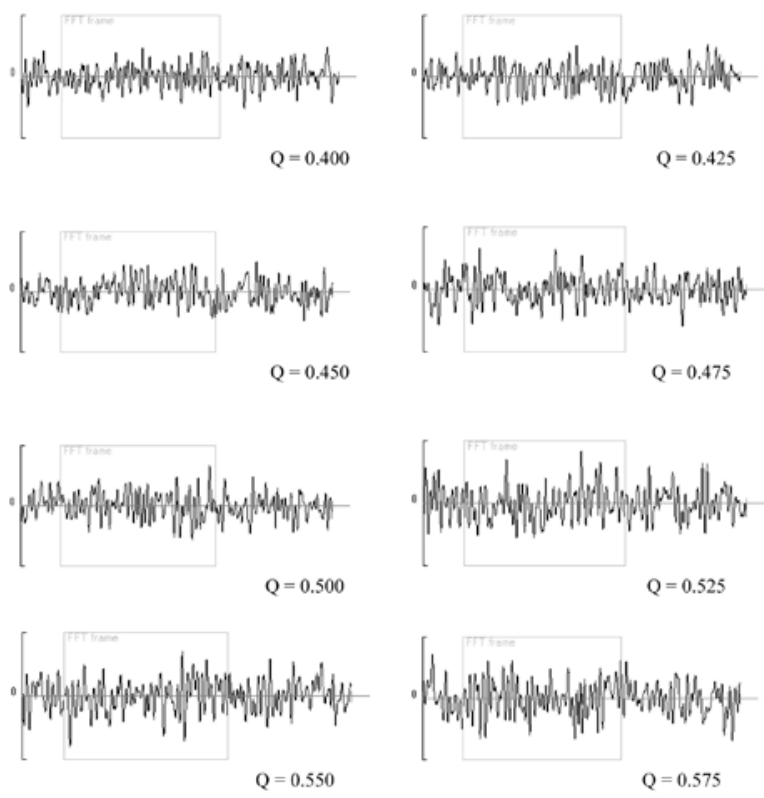

Fig. 5. Simulated response signals for each dynaic pressure

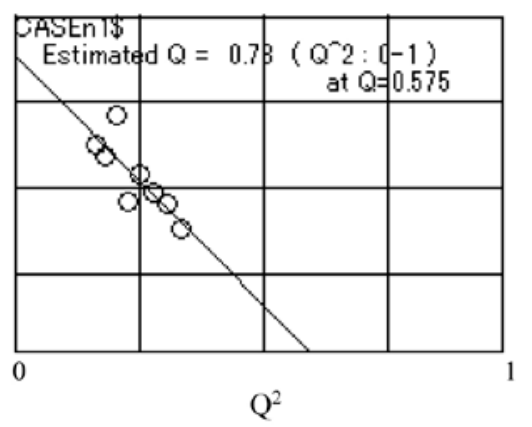

Fig. 6. Wavelet flutter prediction from the simulated signals 


\section{Concluding remarks}

The method using the inverse discrete Fourier transform to simulate the random response of an aeroelastic system has been proposed and demonstrated. It is expected to be utilized for evaluating various methods used to predict flutter and the performance of the active control technique to attenuate the response against the atmospheric turbulence.

\section{References}

Baldelli, D. H.; Ohta, H.; Matsushita, H., et al. 1995. Flutter margin augmentation synthesis using Normalized Coprime Factores Approach, Journal of Guidance, Control, and Dynamics 18(4): 802-811. http://dx.doi.org/10.2514/3.21462

Bisplinghoff, R. L.; Ashley, H.; Halfman, R. L. 1955. Aeroelasticity. Cambridge: Addison-Wesley Pub. Co. Inc. 272 p.

Küssner, H. G. 1940. Genral Airfoil Theory, Luftfahrtforschung 17(11/12): 370-378.

Nishijima, H.; Ueda, T.; Ikeda, T. 2009. A study on reliability of flutter prediction method, in JSASS 51st Conference on Aircraft Structures, 2A12 (in Japanese).

Randall, T. B. 1977. Application of B \& K equipment to frequency analysis. Denmark: Naerum Offset Tryk. 187 p.

Shinozuka, M.; Jan, C. M. 1972. Digital simulation of random processes and its applications, Journal of Sound and Vibration 25(1): 111-128. http://dx.doi.org/10.1016/0022-460X(72)90600-1

Thomson, W. T. 1960. Laplace Transformation. Englewood Cliffs: Prentice-Hall, Inc. 37 p.

Ueda, T.; Iio, M.; Ikeda, T. 2009. Flutter prediction using continuous wavelet transform, Transaction of the Japan Society for Aeronautical and Space Sciences 174: 275-281. http://dx.doi.org/10.2322/tjsass.51.275

Ueda, T. 1983. Doublet Point Method for unsteady lifting surface - part I Subsonic Flow, A report of National Aerospace Laboratory (NAL) TR-781, Tokyo (in Japanese). 